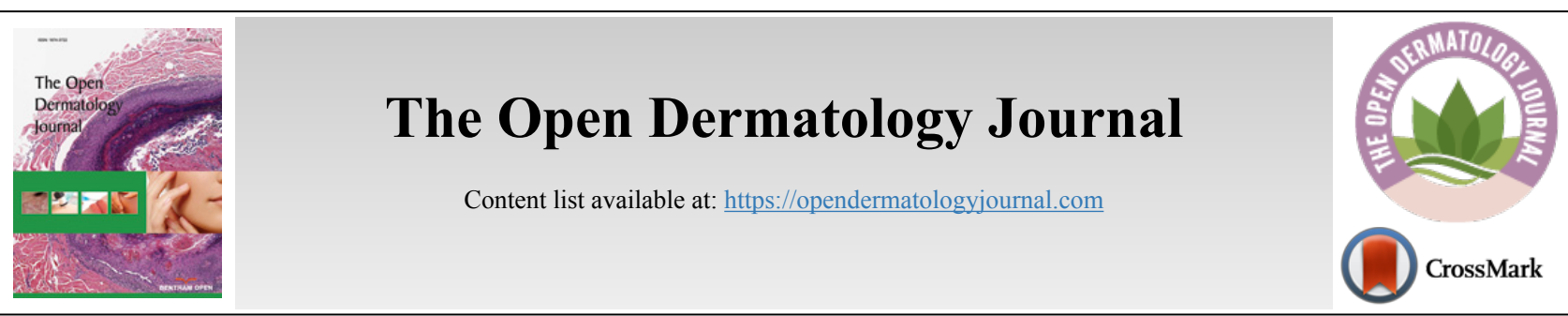

RESEARCH ARTICLE

\title{
Current Status of Computational Intelligence Applications in Dermatological Clinical Practice
}

\author{
Carmen Rodríguez-Cerdeira ${ }^{1,2,3,4, *}$, José Luís González-Cespón ${ }^{1}$ and Roberto Arenas ${ }^{1,3,4,5}$ \\ ${ }^{1}$ Efficiency, Quality, and Costs in Health Services Research Group (EFISALUD), Health Research Institute. SERGAS-UVIGO Vigo, Spain \\ ${ }^{2}$ Dermatology Department, Hospital do Meixoeiro and University of Vigo, Vigo, Spain \\ ${ }^{3}$ European Women's Dermatologic and Venereologic Society (EWDVS), Tui, Spain \\ ${ }^{4}$ Psychodermatology Task Force of the Ibero-Latin American College of Dermatology (CILAD), Buenos Aires, Argentina \\ ${ }^{5}$ Micology Department, Manuel Gea González Hospital, Mexico City, Mexico
}

\begin{abstract}
:
Background:

The yeast infections are increasingly frequent and the correct diagnosis consists of the identification of the yeast fungus, which in our case we are going to refer to the different species of Candida. The prescription of a broad-spectrum antifungal without taking into account the etiological agent, leads to an increase in the resistance to these treatments.

\section{Objective:}

The objective of this work is to differentiate Candida albicans from other Candida species (Candida spp.) By means of digital images obtained from the optical microscope.

\section{Material and Methods:}

It has reviewed about 100 photographs from patients in our consultations.

In this study we will use the microscopic images of the Candida variety to be processed later with the Octave programming language and its image processing package (image-2.8.0).

Results and Discussion:

This system is able to differentiate Candida albicans from the other varieties of Candida such as C. parapsilosis, C. krusei, and C. kefyr with accuracy.

The candida identifier application, which was designed and programmed in Octave, allows identification of candida species by locating certain geometric descriptors, such as the centroid and the surfaces of circular objects within the images. The program was highly effective for the diagnosis of Candida spp. So, we got a sensitivity and specificity above $90 \%$ with the images used.

\section{Conclusion:}

The results that we obtain from the Candida spp. identifier system that opens the way to be able to work with images obtained from the optical microscope.
\end{abstract}

Keywords: Candida spp, Octave, Image processing, Candydos program, Computational intelligence, Clinical practice, Candida infections.

\begin{tabular}{|l|l|l|r|} 
Article History & Received: December 02, 2019 & Revised: February 10, 2020 & Accepted: February 11, 2020
\end{tabular}

\section{INTRODUCTION}

Intelligent computer systems already provide support to healthcare professionals.

\footnotetext{
Address correspondence to this author at the Department of Dermatology, Hospital do Meixoeiro and University of Vigo, Vigo, Spain; Tel: 0034600536114; E-mail: carmencerdeira33@gmail.com
}

Computational intelligence has been widely researched and applied owing to its ability to cope with large amounts of clinical data and uncertain information [1]. Computational intelligence uses algorithms based on biological data, primarily on neuronal functioning. The three pillars on which computational intelligence is based are neural networks, genetic algorithms, and fuzzy systems [2]. Neural networks are algorithms employed for function approximation or classi- 
fication problems. They include supervised, unsupervised, and reinforcement learning [3].

Constant progress is being made on computational intelligence to improve the prevention, diagnosis, and treatment of diseases in general, and mycosis in particular [4].

The yeast Candida albicans is the most prevalent pathogenic Candida species (spp.), although the increase in immunocompromised patients has been accompanied by an increase in the diversity of pathogenic strains found as etiological agents of fungal infections $[5,6]$.

The most typically used method to identify Candida spp. is the chromogenic culture medium. An analysis of the growth facilitates the determination of the purity of the colonies, and the identification of Candida spp [7].

The samples used by us are from this culture medium and were later imaged under an optical microscope. After reviewing several images, we have selected $C$. albicans, $C$. parapsilosis, C. krusei, and C. kefyr for our project. The images used in the elaboration of the program correspond to Figs. (1-7).

\section{MATERIALS AND METHODS}

\subsection{Material}

Approximately 100 photographs primarily from the author's patients were reviewed.

In this study, microscopic images of the Candida species were used and subsequently processed using the Octave programming language and its image processing package (image-2.8.0). The interface was obtained through Electron's libraries, based on JavaScript and HTML.

\subsection{Description of the Method}

Development of programs for the detection of Candida species

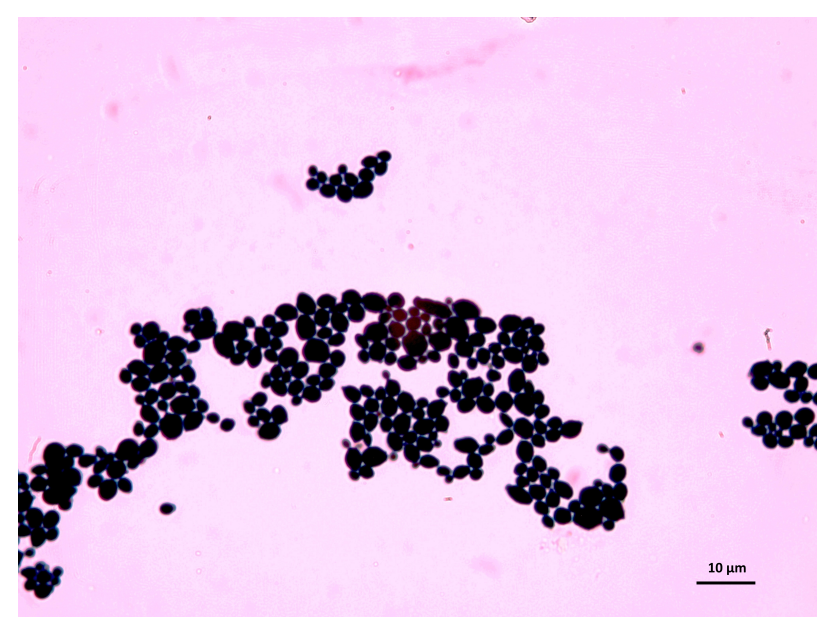

Fig. (1). Candida albicans.

As the characteristics of the different species of Candida are known, we applied digital image processing with Octave. We performed an adaptation of the diagnostic methods of the
Candida and their subsequent study under a microscope for the classification of the different species Most of these methods have typical concepts, such as the variety of colors, asymmetry in their internal forms, and abrupt and irregular edges, and are used to identify elements of the said fungi under the optical microscope. These are the primary concepts that are analyzed in the programs performed, and are referred to as the "Candida Identifier."

\section{OBJECTIVES}

- The objective of this work is to differentiate Candida albicans from other Candida spp. with an accuracy of approximately $70 \%$.

This general objective is divided into more specific objectives as follows:

- Establish the spaces of color and form that will be used in this work.

- Define the necessary programs, in Octave environment, such that they can recognize the different species of Candida

- Develop a rapid, easy, and economical technique for identifying Candida spp. that is easily implementable and manageable even by primary care physicians.

\section{RESULTS AND DISCUSSION}

(1) The identification algorithm was performed using the following steps:

- Load the images

- Go from RGB to grayscale

- Binarization of the image

- Tag pixel regions to establish neighborly relations

- Perform geometric measurements to select descriptors

- Pattern determination

- Choice of patterns to differentiate the species of Candida (Candida spp.)

(2) Later, we will perform the stages of the model comprising the following:
(A) Identification and training criteria
(B) Testing
(C) Validation
(D) Implementation of the algorithm through Octave and program development

(E) Interface design

\subsection{Identification and Training Criteria}

Subsequently, with part of the program code, we seek to identify the circularity of each element, which is stored in the circularities variable. The results are weighted with an arbitrary threshold to define the number of circular elements of the images, which is stored in the variable roundObjects. These characteristics allow us to differentiate the species studied. For 
example, C. albicans exhibits circular elements resembling C. kefyr. Meanwhile, C. krusei exhibits more linear elements.

To calculate of the circularity of rounded objects we use the follow program code in Table $\mathbf{1}$.

Table 1. Determination of the circumscribed circumference from the centroid of the silhouette.

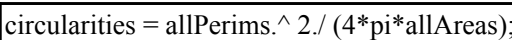

Weighting to discriminate circular components:

roundObjects $=$ find $($ circularities $<4)$;

Table 2. Confusion matrix with the different Candida spp.

\begin{tabular}{|c|c|c|c|c|c|}
\hline \multirow{2}{*}{ Comparison } & \multicolumn{5}{|c|}{ Identifier Value } \\
\hline \multirow{3}{*}{ Real Value } & $\mathrm{x} / \mathrm{x}$ & C. albicans & C. krusei & C. kefyr & \multicolumn{1}{c|}{ C. parapsilosis } \\
\cline { 2 - 6 } & C. albicans & 100 & 0 & 0 \\
\cline { 2 - 6 } & C. krusei & 0 & 90 & 10 & 0 \\
\cline { 2 - 6 } & C. kefyr & 0 & 10 & 0 \\
\cline { 2 - 6 } & C. parapsilosis & 0 & 0 & 0 \\
\hline
\end{tabular}

Table 3. Results of confusion matrix with $C$. albicans, C. krusei, C. kefyr \& C. parasilopsis

Hit rate $=$ Identification $/$ Actual value

Hits C. albicans: $100100,100 \%$

Hits C. krusei: $90100,90 \%$

Hits C. Kefyr: 90100, 90\%

Hits C. parapsilosis: $100100,100 \%$

The images used in the elaboration of the program correspond to figures $1,2,3$, and 4 .

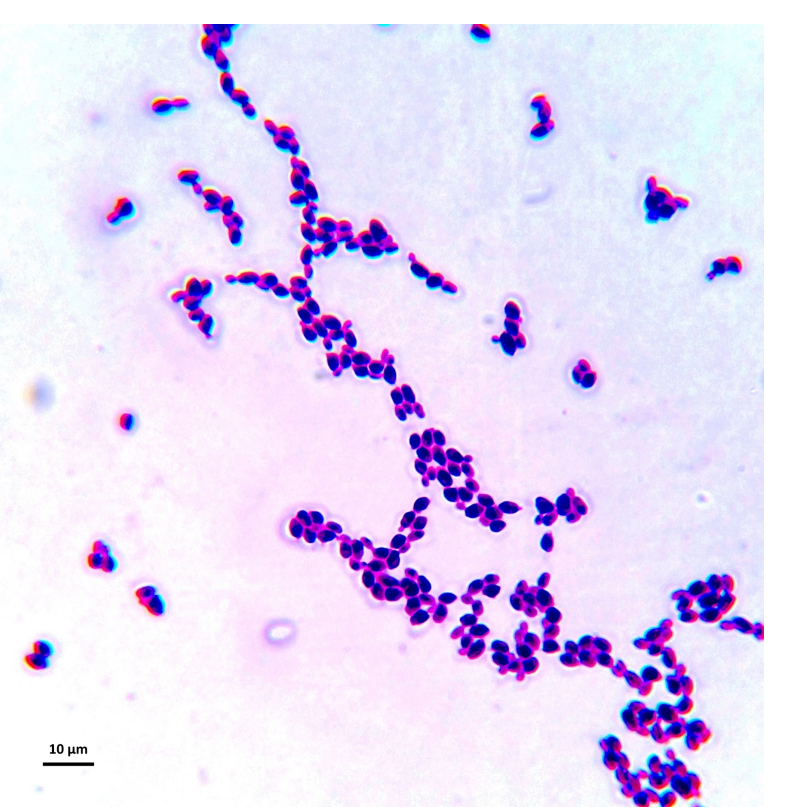

Fig. (2). Candida Kefyr. 


\subsection{Implementation of the Algorithm Through Octave and Development of the "Candydos" Program}

The program has implemented the image recognition algorithm, using the leeImage function. In its first line, the image library of Octave is loaded. This library contains the classes and functions that allow for the processing of mushroom images (Table 4)

\subsection{Interface from Database of Java Script Libraries}

Finally, as Octave does not have a method to generate the GUI, the interface has been implemented through the Electron framework, which is developed in JavaScript, and HTML (Table 5).

\section{Table 4. Recognition algorithm (leeImage function).}

function [identificador] = leeImagen(file)

pkg image load;

$\%$ This part reads the file of the image that will be classified by the $\%$ recognition program.

RGB = imread (file);

$\%$ The RGB image is passed in grayscale, that is, the original image with its component $\%$ Red, Green, and Blue

$\mathrm{I}=\operatorname{rgb} 2$ gray $(\mathrm{RGB})$;

$\%$ In this stage, the image that is in ray scale is binarized. This operation passes $\%$ first from the format uint 8 to double. Having this format, the scale of gray $\%$ is compared with the threshold 0.2 . Values above 0.2 become! (white) and below\% 0 (black).

$\mathrm{BW}=\mathrm{im} 2 \mathrm{bw}(\mathrm{I}, 0.2)$;

$\%$ The image is displayed

$\%$ imshow (BW)

$\%$ The bwlabel command labels the binary image, such that it establishes contiguous (neighboring) zones to determine the regions.

abeledImage $=$ bwlabel $(\mathrm{BW})$;

$\%$ The regionprops function allows for the determination of the geometric descriptors of the figures obtained, after binarizing the images. The areas are found, as well as the perimeters that are essential\% to initially identify the circular regions. This descriptor

allows the $\%$ fungi types to be differentiated. Thus, for example, the circular area of a Candida $\%$ albicans fungus differentiates it from a parapsilosis fungus $\%$.

measurements $=$ regionprops (labeledImage, 'Area', 'Perimeter');

allAreas $=$ [measurements.Area $]$;

allPerims $=[$ measurements.Perimeter $]$;

circularities $=$ allPerims. ${ }^{\wedge} 2 . /(4 *$ pi $*$ allAreas $)$;

blobMeasurements $=$ regionprops (labeledImage, 'BoundingBox', 'Area');

allBlobAreas $=$ [blobMeasurements.Area $]$;

$\%$ Find objects that have "round" values of circularities.

roundObjects $=$ find $($ circularities $<4) ; \%$ Whatever value you want.

$\%$ Compute new binary image with only the round objects in it.

binaryImage $=$ ismember (labeledImage, roundObjects) $>0$;

identificador $=$ find $($ circularities $<4 \&$ allBlobAreas $>10000)$;

$\%$ figure(2)

\%imshow(binaryImage);

Endfunction

$\%$ In this stage, the images that are being loaded to the application $\%$ are simply classified according to the identifier that has been defined for the

fungi in the function.

$\%$ clear all; clc;

\# not a function file:

pkg image load;

file = 'ima.jpg';

[identifier] = readImage (file);

if length (identifier) $==1 \& \&($ identifier $<10)$

disp ("The fungus is Candida parapsilosis")

elseif length (identifier) $==1 \& \&$ (identifier $>20) \& \&($ identifier $<30)$

disp ("The fungus is Candida albicans")

elseif length (identifier> 100)

disp ("The fungus is Candida kefyR")

else

disp ("The fungus is Candida krusei")

endif

fflush.stdout () 
Table 5. Java Script libraries and frameworks.

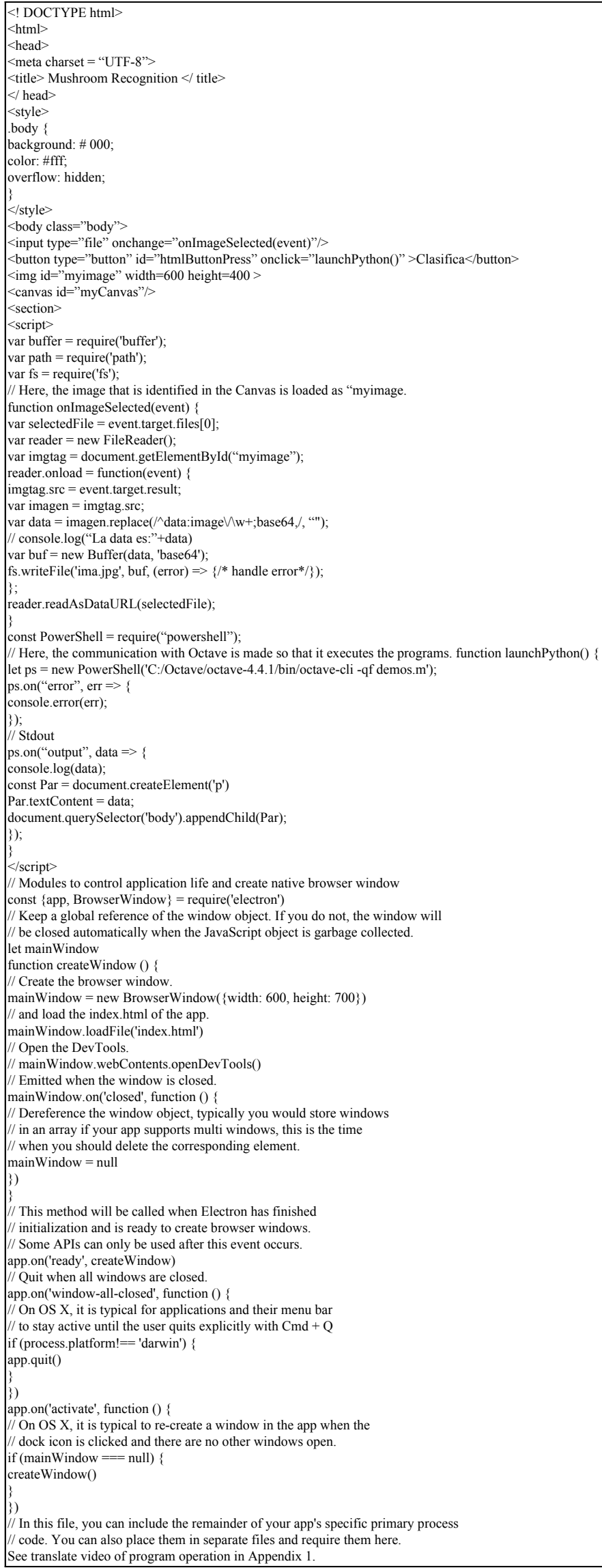




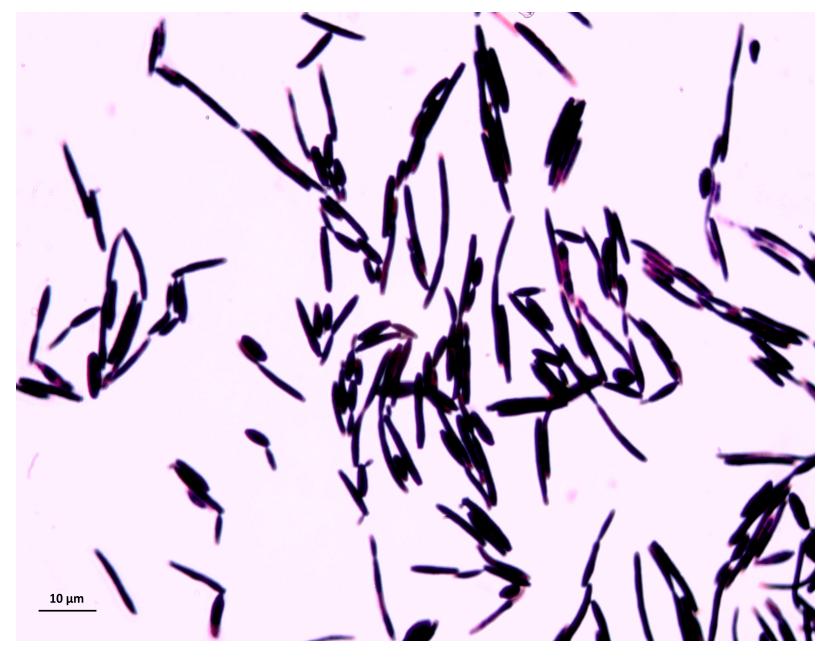

Fig. (3). Candida krusei.

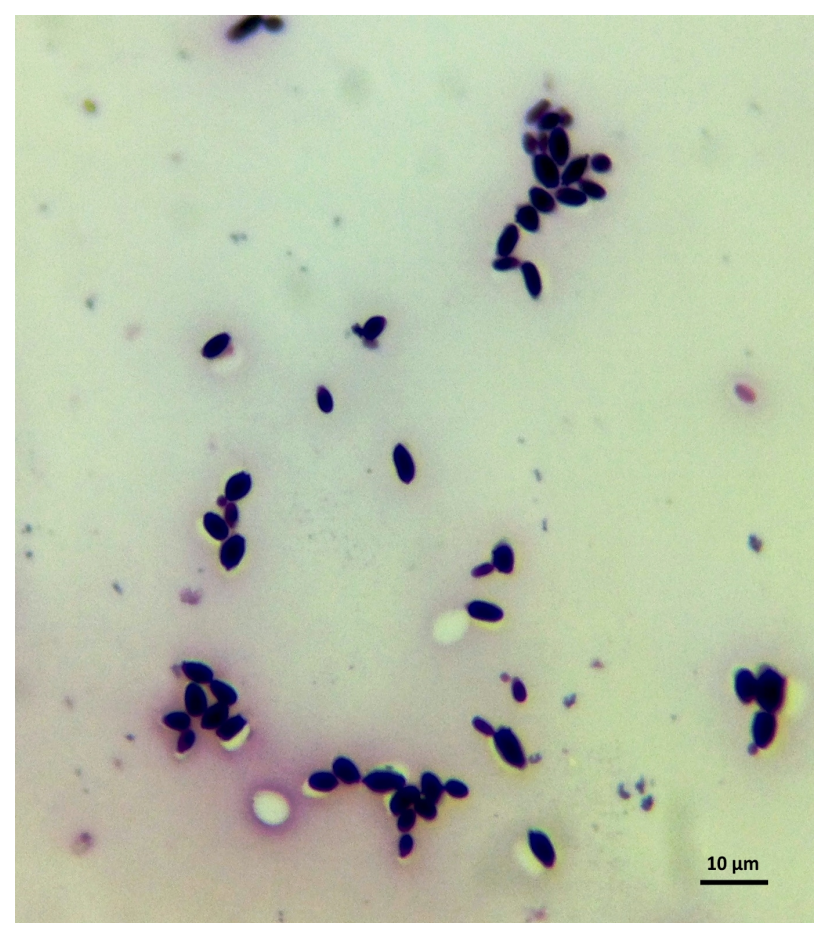

Fig. (4). Candida parapsilosis.

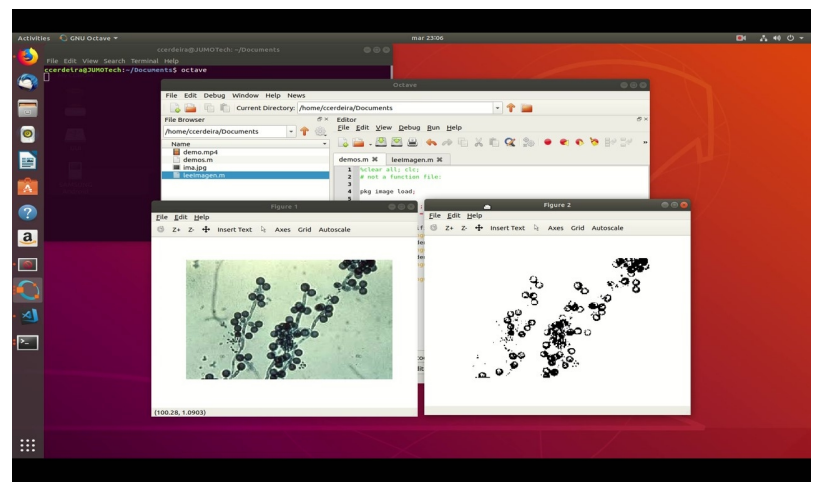

Fig. (5). The RGB image (original image) is passed to grayscale and subsequently to its binary form.

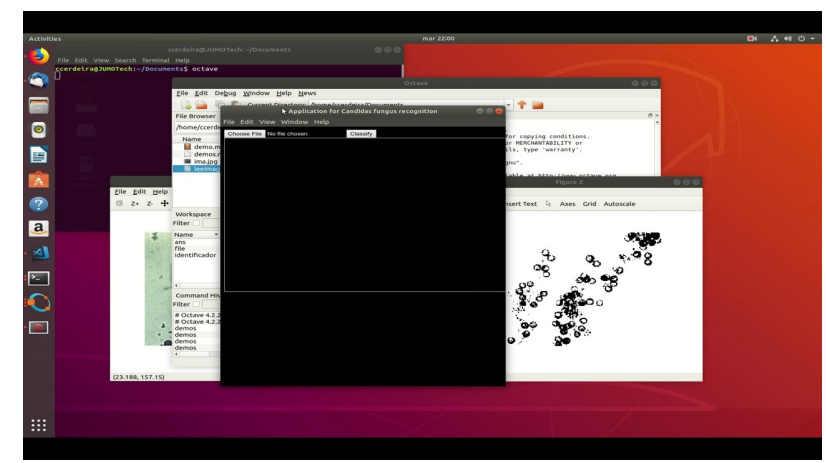

Fig. (6). Application interface: First step of the Candida recognition process.

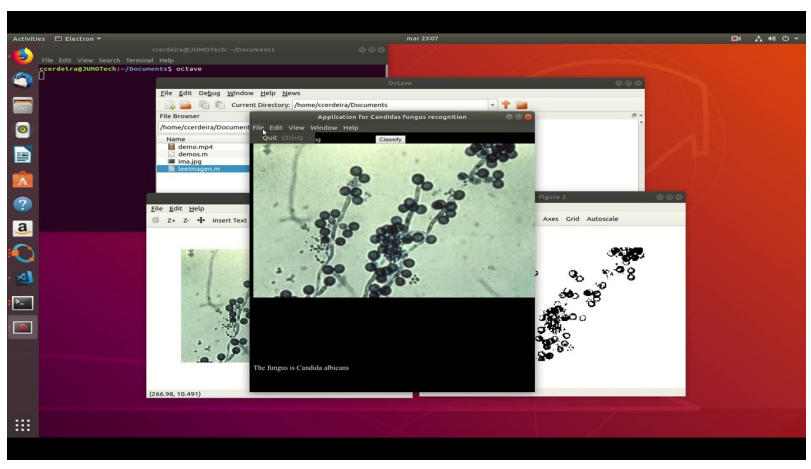

Fig. (7). Step two of the Candida recognition process and step three, recognition by "candydos" of Candida spp. In this case, the one identified with correction was Candida albicans.

\section{DISCUSSION}

A major difficulty was in obtaining a large sample and images of the Candida spp. used with similar characteristics, because the time of planting and the technique vary according to its shape and size. The system cannot change the size of the images during the entire process [9].

Another difficulty that also influences the classification of the image is the image quality $[10,11]$.

The more the noise or the poorer the image quality, the less precision the edge mapping process will have [12].

Further, the less accuracy on the border mapping process, the worse is the performance on the classification process [13].

In the literature, we did not find models that were used to recognize Candida spp. However, we found references bout the artificial neural networks to calculate antifungal activity against C. albicans. Furthermore, there are references to other cutaneous diseases [14 - 16] such as melanoma [17].

Hence, we established a goal. The objective was to reach a diagnostic accuracy of at least $80 \%$. The model proposed by Manousaki et al. [18] provided an accuracy of $89.4 \%$. The model for pre-diagnostic digital imaging developed by Christensen et al. [19] presented an accuracy of $77 \%$. Our model is superior in accuracy to those mentioned above.

The final prediction model developed exhibits an accuracy of almost $90 \%$ and will thereby represent an improvement in 
diagnostic accuracy. Despite the different appearances and qualities of the images in the image database, we could develop a prediction model with accuracy better than that expected from an experienced dermatologist [20].

The algorithms are thereby stable and not dependent on the quality of the image /under inspection. These new tools can also be utilized in telemedicine, where images can be uploaded to an automated web-based database and subsequently analyzed [21].

\section{CONCLUSION}

We designed and programmed the "Candida identifier application" in Octave. It allowed the identification of the Candida species by locating certain geometric descriptors, such as the centroid and the surfaces of the circular objects that comprise the images. This system could differentiate the $C$. albicans from other varieties of Candida such as C. Kefyr, $C$. parapsilosis, and C. krusei, with accuracy. The analysis by the system of these descriptors allowed us to identify the Candida species in more than $90 \%$ of the cases. The sensitivity and specificity that were above $90 \%$ indicated a high diagnostic efficacy that was rather large to be the first version of a diagnostic program.

The application was highly precise in the identification based on the forms; however, it did not exhibit the same features in color-based identification. This element typically presents variations in the data, thus rendering identification difficult. We should acquire more copious databases to implement more precise identification methods, such as deep learning algorithms for images; however, the recognition of information in three different areas was satisfactory, as it allowed us to determine and classify injuries within the medical margins.

Finally, we emphasize that the objectives set at the beginning of the project were achieved. The results that we obtained from the "Candida identifier system" paved the way for handling images obtained from an optical microscope. This implies a wider range of materials can be used and specialized personnel economy (mainly in developing countries), and better service from the patient's perspective.

\section{AUTHORS' CONTRIBUTORS}

"Candydos" program. CRC designed and implemented the algorithm for "candydos" through Octave, as well as developed its program.

JLGC has contributed to create the algorithms of the program

RA has provided and selected Candida spp.

\section{ETHICS APPROVAL AND CONSENT TO PARTI- CIPATE}

This study was approved by the Ethics and Research Committee of Hospital General Manuel Gea Gonzalez, Estado de México, Mexico. (Approval Number: OF /No 145001022 151/CCEEIS/016/2018).

\section{HUMAN AND ANIMAL RIGHTS}

No Animals were used in this research. All human research procedures followed were in accordance with the ethical standards of the committee responsible for human experimentation (institutional and national), and with the Helsinki Declaration of 1975, as revised in 2013.

\section{CONSENT FOR PUBLICATION}

Written informed consent was obtained from patients for the use of the fungal isolates and the publication of the cases details.

\section{AVAILABILITY OF DATA AND MATERIAL}

$\square \square$ The data that support the findings of this study are available from the corresponding author [C.C] upon request.

\section{FUNDING}

The authors received no financial support for the research, authorship, and/or publication of this article.

\section{CONFLICT OF INTEREST}

The authors declare no conflict of interest, financial or otherwise.

\section{ACKNOWLEDGEMENTS}

Declared none.

\section{SUPPLEMENTARY MATERIAL}

Supplementary material is available on the publishers web site along with the published article.

\section{APPENDIX 1}

\section{DESCRIPTION OF THE VIDEO CONTENT}

The video includes the creation of the algorithm, and the diagnosis and operation of the program:

First, "\%" passes the RGB image in grayscale, that is, the original image with its "\%" red, green, and blue components (binarization of the image).

Subsequently, "\%" classifies the images that are loaded to the application\% according to the identifier that has been defined for the fungi in the "readImage" function

Finally, how the different species of Candida are presented and how the program identifies them with an accuracy of at least $90 \%$ are observed.

Steps of the video

1

00: 00: 02,193 -> 00: 00: 03,003

$<\mathrm{i}>$ Program code showing the results $</ \mathrm{i}>$

2

00: 00: 04,193 -> 00: 00: 06,003

$<\mathrm{i}>$ Code of the program that analyzes the images $</ \mathrm{i}>$ 
3

00: 00: 07,193 -> 00: 00: 11,003

$<\mathrm{i}>$ Save and play the program $</ \mathrm{i}>$

4

00: 00: 20,193 -> 00: 00: 24,003

$<\mathrm{i}>$ Real and binarized image $</ \mathrm{i}>$

5

00: 00: 25,193 -> 00: 00: 28,503

$<\mathrm{i}>$ Command window: The fungus is Candida kefyr $</ \mathrm{i}>$

6

00: 00: 44,193 -> 00: 00: 46,003

$<\mathrm{i}>$ Graphic interface $</ \mathrm{i}>$

7

00: 00: 46,993 -> 00: 00: 49,503

$<\mathrm{i}>$ Choose file $</ \mathrm{i}>$

8

00: 00: 52,073 -> 00: 00: 56,003

$<\mathrm{i}>$ Classify: The fungus is Candida albicans $</ \mathrm{i}>$

9

00: 00: 59,193 -> 00: 01: 02,503

$<\mathrm{i}>$ Choose file $</ \mathrm{i}>$

10

00: 01: 04,033 -> 00: 01: 07,021

$<\mathrm{i}>$ Classify: The fungus is Candida kefyr $</ \mathrm{i}>$

11

00: 01: 08,193 -> 00: 01: 09,803

$<\mathrm{i}>$ Choose file $</ \mathrm{i}>$

12

00: 01: 14,173 -> 00: 01: 17,303

$<\mathrm{i}>$ Classify: The fungus is Candida parapsilosis $</ \mathrm{i}>$

\section{REFERENCES}

[1] López-Rubio E, Elizondo DA, Grootveld M, Jerez JM, Luque-Baena RM. Computational intelligence techniques in medicine. Comput Math Methods Med 2015; 2015196976

[http://dx.doi.org/10.1155/2015/196976] [PMID: 25834633]

[2] Haykin S. Neural networks: A comprehensive foundation. New Jersey: Prentice Hall PTR 1998.

[3] Hamet P, Tremblay J. Artificial intelligence in medicine. Metabolism 2017; 69S: S36-40.

[http://dx.doi.org/10.1016/j.metabol.2017.01.011] [PMID: 28126242]

[4] Koulouri A, Kuonen F, Gaide O. Artificial intelligence and the skin specialist. Rev Med Suisse 2019; 15(644): 687-91. [PMID: 30916908]

[5] Hedayati MT, Tavakoli M, Zakavi F, et al. In vitro antifungal susceptibility of Candida speciesisolated from diabetic patients Rev Soc Bras Med Trop 2018; 51(4): 542-5.

[http://dx.doi.org/10.1590/0037-8682-0332-2017] [PMID: 30133642]

[6] Şular FL, Szekely E, Cristea VC, Dobreanu M. Invasive fungal infection in romania: Changing incidence and epidemiology during six years of surveillance in a tertiary hospital. Mycopathologia 2018; 183(6): 967-72.

[http://dx.doi.org/10.1007/s11046-018-0293-2] [PMID: 30168077]

[7] Ouanes A, Kouais A, Marouen S, Sahnoun M, Jemli B, Gargouri S. Contribution of the chromogenic medium CHROMagar((R)Candida in mycological diagnosis of yeasts. J Mycol Med 2013; 23(4): 237-41. [http://dx.doi.org/10.1016/j.mycmed.2013.07.058] [PMID: 24161925]

[8] GitHub, Inc. [US]. Electron packge/ electron-quick-start https://github.com/electron/electron-quick-start2018.

[9] Colling R, Pitman H, Oien K, et al. Artificial intelligence in digital pathology: A roadmap to routine use in clinical practice. J Pathol 2019; 249(2): 143-50

[http://dx.doi.org/10.1002/path.5310] [PMID: 31144302]

[10] Aractingi S, Pellacani G. Computational neural network in melanocytic lesions diagnosis: Artificial intelligence to improve diagnosis in dermatology? Eur J Dermatol 2019; 29(S1): 4-7. [http://dx.doi.org/10.1684/ejd.2019.3538] [PMID: 31017580]

[11] Wu M, Yan C, Liu H, Liu Q. Automatic classification of ovarian cancer types from cytological images using deep convolutional neural networks. Biosci Rep 2018; 38(3)BSR20180289

[http://dx.doi.org/10.1042/BSR20180289] [PMID: 29572387]

[12] Chang HY, Jung CK, Woo JI, et al. Artificial Intelligence in Pathology. J Pathol Transl Med 2019; 53(1): 1-12.

[http://dx.doi.org/10.4132/jptm.2018.12.16] [PMID: 30599506]

[13] Ware C. Perception for Design (Interactive Technologies). 2nd ed. San Francisco, U.S.A: Morgan Kaufmann 2004.

[14] Hogarty DT, Su JC, Phan K, et al. Artificial intelligence in dermatology-where we are and the way to the future: A review. Am J Clin Dermatol 2019.

[http://dx.doi.org/10.1007/s40257-019-00462-6] [PMID: 31278649]

[15] Aggarwal SLP. Data augmentation in dermatology image recognition using machine learning. Skin Res Technol 2019; 25(6): 815-20. [http://dx.doi.org/10.1111/srt.12726]

[16] Min S, Kong HJ, Yoon C, Kim HC, Suh DH. Development and evaluation of an automatic acne lesion detection program using digital image processing. Skin Res Technol 2013; 19(1): e423-32.

[http://dx.doi.org/10.1111/j.1600-0846.2012.00660.x] [PMID: 22891680]

[17] Magalhaes C, Mendes J, Vardasca R. The role of AI classifiers in skin cancer images. Skin Res Technol 2019; 25(5): 750-7.

[http://dx.doi.org/10.1111/srt.12713] [PMID: 31106913]

[18] Manousaki AG, Manios AG, Tsompanaki EI, et al. A simple digital image processing system to aid in melanoma diagnosis in an everyday melanocytic skin lesion unit: A preliminary report. Int J Dermatol 2006; 45(4): 402-10.

[http://dx.doi.org/10.1111/j.1365-4632.2006.02726.x] [PMID: 16650167]

[19] Christensen JH, Soerensen MB, Linghui Z, Chen S, Jensen MO. Prediagnostic digital imaging prediction model to discriminate between malignant melanoma and benign pigmented skin lesion. Skin Res Technol 2010; 16(1): 98-108.

[http://dx.doi.org/10.1111/j.1600-0846.2009.00408.x] [PMID: 20384888]

[20] Ryan P, Luz S, Albert P, Vogel C, Normand C, Elwyn G. Using artificial intelligence to assess clinicians' communication skills. BMJ 2019; 364: 1161.

[http://dx.doi.org/10.1136/bmj.1161] [PMID: 30659013]

[21] Kuziemsky C, Maeder AJ, John O, et al. Role of Artificial Intelligence within the Telehealth Domain. Yearb Med Inform 2019; 28(1): 35-40. [http://dx.doi.org/10.1055/s-0039-1677897] [PMID: 31022750]

This is an open access article distributed under the terms of the Creative Commons Attribution 4.0 International Public License (CC-BY 4.0), a copy of which is available at: https://creativecommons.org/licenses/by/4.0/legalcode. This license permits unrestricted use, distribution, and reproduction in any medium, provided the original author and source are credited. 